\title{
Temporal Discretization Algorithms for the Continuity Equation of the One-dimensional Shallow Water Model
}

\author{
Hidekazu Yoshioka ${ }^{1}$, Koichi Unami ${ }^{2}$ and Masayuki Fujihara ${ }^{3}$
}

\begin{abstract}
Cross-sectionally averaged one-dimensional shallow water equations (1-D SWEs) serve as a fundamental tool in simulating open channel flows. Accurate numerical simulation of transient flows is crucial for practical analysis of mass and momentum transport phenomena in open channels. Temporal discretization algorithm is the one of the most influential factors that controls accuracy, stability and computational efficiency of numerical simulation. This paper presents a new numerical method for the 1-D SWEs, referred to as SElective LUmping Method (SELUM), which is an intermediate method between the Finite Element/Volume Method (FEVM) and Dual Finite Volume Method (DFVM) previously developed by the authors. The SELUM selectively lumps the mass matrix of the continuity equation so that stable numerical solutions are obtained even under severe flow conditions where the FEVM or DFVM fail. An approximate mass matrix inversion method is incorporated into the SELUM to improve computational efficiency while not to degrade accuracy and stability. Numerical simulations of idealized and experimental problems are carried out to demonstrate its advantages over the existing numerical methods.
\end{abstract}

Keywords: Shallow water equations; Finite element method; Finite volume method; Selective lumping algorithm

\section{Introduction}

Shallow water equations (SWEs), a system of nonlinear hyperbolic partial differential equations (PDEs) with source terms, serve as a fundamental tool for practical analysis of mass and momentum transport phenomena in surface water bodies. Advantages of the SWEs over the multi-dimensional and non-hydrostatic models (Boscheri et al., 2012; Dumbser, 2013) are the low-dimensionality in reasonably describing water flows. On the other hand, their disadvantages are the hydrostatic pressure assumption that may lead to erroneous results for the problems where dispersive surface water dynamics play crucial roles and the uni-variate assumption of water surface, indicating that they cannot simulate undular water surface variations and large water surface deformations such as wave breakings. Nevertheless, the SWEs work more effective than the non-hydrostatic models for the problems with a sufficiently large horizontal scale in which vertical dynamics of water flows is not of basic importance. Furthermore, in spite of the bold simplification employed in describing the dynamics of water flows, solutions to the SWEs are reasonably accurate for a wide range of important complex flows in engineering applications (Begnudelli and Sanders, 2007; Malleron et al., 2011; Hodges, 2013).

Water flows along a channel with a dominant flow direction are effectively modeled with the cross-sectionally averaged one-dimensional shallow water equations (1-D SWEs) in which a channel is identified with a Jordan curve

\footnotetext{
${ }^{1}$ Doctoral Student, Research Fellow of Japan Society for the Promotion of Science, Graduate School of Agriculture, Kyoto University, Kitashirakawa-Oiwake-cho, Sakyo-ku, Kyoto 606-8502, Japan (Corresponding Author): E-mail: yoshih@kais.kyoto-u.ac.jp

${ }^{2}$ Associate Professor, Graduate School of Agriculture, Kyoto University, Kitashirakawa-Oiwake-cho, Sakyo-ku, Kyoto 606-8502, Japan

${ }^{3}$ Professor, Graduate School of Agriculture, Kyoto University, Kitashirakawa-Oiwake-cho, Sakyo-ku, Kyoto 606-8502, Japan
}

(Cunge et al., 1980; Szymkiewicz, 2010). Resolution of the 1-D SWEs requires the use of a numerical method in general because they have analytical solutions only for the cases under simplified conditions (Stoker, 1957; Delestre et al., 2013). Many numerical methods have been developed for the 1-D SWEs. Most of them utilize the finite volume method (FVM) or finite element method (FEM). The FVM is based on a local integral formulation analogous to the Gauss-Green formula that ensures mass and momentum conservation properties (Roe, 1981; Osher and Solomon, 1982). The FEM is not necessary conservative but has higher phase and amplitude accuracy than the FVM (Christon et al., 2004; Voth et al., 2004). Other recently developed numerical methods, such as the discontinuous Galerkin FEM (Dumbser and Casulli, 2013; Tumoro et al., 2013), kinetic interpretation method (Goutal and Santie-Marie, 2011; Roggensack, 2012), residual distribution method (Ricchiuto and Bollermann, 2009; Sármány et al., 2013) and Lagrangian particles method (Vacondio et al., 2011; Xia et al., 2013) have also been applied to the 1-D SWEs. The authors developed numerical methods that utilize staggered spatial discretization procedures to efficiently solve the 1-D SWEs in single channels and channel networks: the Finite Element/Volume Method (FEVM) (Unami and Alam, 2012) and the Dual Finite Volume Method (DFVM) (Yoshioka and Unami, 2012). Both of the numerical methods have extensively been applied to a number of test and real problems demonstrating their sufficiently high accuracy. Advantages of the FEVM and DFVM over the conventional numerical methods are the use of compact computational stencils to accurately capture flows and to efficiently and consistently deal with singularities, such as junctions and channel bends.

An essential difference between the DFVM and FEVM is the discretization procedures applied to the temporal term 
of the continuity equation: the term in the FEVM associates a positive-definite mass matrix with positive non-diagonal entries, while that of in the DFVM associates a diagonal one (Mishev, 1998). The use of the diagonal mass matrix in the DFVM, which is inverted analytically without numerical procedures, contributes to its high computational efficiency (Yoshioka and Unami, 2012).

Although the FEVM and DFVM are comparably accurate for the flows without transitions and wet and dry interfaces, their remarkable limitations in severe flow conditions were revealed in Yoshioka et al. (2012). According to them, the FEVM is not capable of simulating some flows with wet and dry interfaces in non-rectangular channels. An example is a dry dam break flow in a triangular channel (Sanders, 2001). On the other hand, the DFVM fails to compute solutions with a sudden transition from supercritical to subcritical where the flow hits a solid wall. An example is a hydraulic bore propagating upstream resulting from a sudden closure of the downstream-end of an open channel (Unami and Alam, 2012). In real problems, both kinds of water flows are frequently encountered as indicated in the literatures (Boudarias and Gerbi, 2007; Ono et al., 2011). Improvement of these drawbacks therefore contributes to develop a versatile numerical method that handles wider range of problems.

The purpose of this paper is to develop a new numerical method for the 1-D SWEs (SELUM: SElective LUmping Method) that incorporates the advantages of the FEVM and DFVM. This paper focuses in particular on application of the SELUM to the 1-D SWEs in single channels. Discretization of the SELUM is similar to those of the DFVM and FEVM except for the treatment of the temporal term of the continuity equation. The term is selectively lumped on the basis of local flow conditions to handle the flows that the FEVM or DFVM fails. An approximate mass matrix inversion method of Guermond and Pasuquetti (2013) is utilized in order to improve computational efficiency while maintaining accuracy and stability. The SELUM is verified with test and real problems to demonstrate its versatility and advantages over the FEVM and DFVM.

The remainder of this paper is organized as follows. A concise introduction for the 1-D SWEs is given in Section 2. Spatial and temporal discretization procedures of the SELUM are presented in Section 3. The SELUM, FEVM and DFVM are applied to test and real problems in Section 4. Section 5 provides conclusions and future perspectives of this study.

\section{Shallow water equations (1-D SWEs)}

The 1-D SWEs (Unami and Alam, 2012) for water flows along a single channel with arbitrary cross-sectional shapes consist of the continuity equation

$$
T \frac{\partial \eta}{\partial t}+\frac{\partial Q}{\partial x}=0
$$

and the momentum equation

$$
\frac{\partial Q}{\partial t}+\frac{\partial F}{\partial x}+g A\left(\frac{\partial \eta}{\partial x}+S_{\mathrm{f}}\right)=0
$$

with the momentum flux

$$
F=\frac{\beta Q^{2}}{A},
$$

where $t$ is the time, $x$ indicates the 1-D abscissa taken along the channel, $A$ is cross-sectional area of flow, $T$ is the top width of the water surface, $Q$ is the discharge, $\beta$ is the momentum coefficient taken as 1 throughout this paper, $g$ is the gravitational acceleration, $S_{\mathrm{f}}$ is the friction slope given by the Manning's formula (Yen, 2002)

$$
S_{\mathrm{f}}=\frac{n_{\mathrm{M}}^{2} P^{4 / 3}}{A^{10 / 3}} Q|Q|
$$

where $n_{\mathrm{M}}$ is Manning's coefficient and $P$ is the wetted perimeter.

\section{Selective lumping method (SELUM)}

This section describes spatial and temporal discretization procedures used in the SELUM.

\subsection{Spatial discretization}

Analogous to the FEVM and DFVM, the SELUM divides a channel into linear elements bounded by two nodes. The water surface elevation $\eta$ and the discharge $Q$ are the dependent variables in the SELUM, which are distributed to the nodes and elements, respectively. Total numbers of the nodes and elements are denoted as $n_{\mathrm{n}}$ and $n_{\mathrm{e}}\left(=n_{\mathrm{n}}-1\right)$, respectively. Eq.(1) is solved with the standard Galerkin FEM with continuous linear basis, while Eq.(2) with a cell-centered FVM, both of which are similar to those of the FEVM and DFVM (Unami and Alam, 2012; Yoshioka and Unami, 2012). Application of the SELUM to Eqs.(1) and (2) leads to the ordinary differential equations (ODEs) of the forms

$$
\mathbf{M} \frac{\mathrm{d} \boldsymbol{\eta}}{\mathrm{d} t}=\mathbf{a}
$$

and

$$
\mathbf{I} \frac{\mathrm{d} \mathbf{Q}}{\mathrm{d} t}=\mathbf{b}
$$

respectively where $\mathbf{M}=\left[M_{i, j}\right]$ is the $n_{\mathrm{n}} \times n_{\mathrm{n}}-$ dimensional mass matrix, $\mathbf{I}=\operatorname{diag}\{1,1, \ldots, 1\}$ is the $n_{\mathrm{e}} \times n_{\mathrm{e}}$-dimensional identity matrix, $\boldsymbol{\eta}=\left[\eta_{i}\right]$ is the $n_{\mathrm{n}}$-dimensional vector of the nodal $\eta, \mathbf{Q}=\left[Q_{i}\right]$ is the $n_{\mathrm{e}}$-dimensional vector of the elemental $Q, \mathbf{a}=\left[a_{i}\right]$ and $\mathbf{b}=\left[b_{i}\right]$ are the $n_{\mathrm{n}}-$ and $n_{\mathrm{e}}$-dimensional vectors explicitly evaluated at each time step.

\subsection{Temporal discretization}

According to Yoshioka et al. (2012), application of a full-lumping to the mass matrix $\mathbf{M}$, analogous to the DFVM, effectively resolves wet and dry interfaces where 
the flows are supercritical. However, the DFVM is unstable for supercritical flows on wet beds that hit solid walls where transitions from supercritical to subcritical occur. On the other hand, the FEVM is suitable for solving this kind of flows accurately reproducing the transitions. These findings suggest the development of a selective numerical algorithm that lumps $\mathbf{M}$ in supercritical flows but does not for subcritical and critical flows.

The SELUM lumps the mass matrix $\mathbf{M}$ of Eq.(5) on the basis of local flow conditions. The new lumping procedure for $\mathbf{M}$ is developed with an efficient, approximate mass matrix inversion method of Guermond and Pasuquetti (2013) whose higher accuracy over the fully-lumped counterpart for hyperbolic PDEs was demonstrated in the literature. The method is computationally efficient compared with the conventional iterative matrix inversion-based ones and has effectively been applied to nonlinear hyperbolic PDEs of mass and momentum transport phenomena (Marras et al., 2013; Yoshioka et al., 2013c). In the SELUM, the product of the vector $\mathbf{a}$ and the inverse $\mathbf{N}=\left[N_{i, j}\right]$ of the mass matrix $\mathbf{M}$ is evaluated as

$$
\sum_{j=1}^{n_{\mathrm{n}}} N_{i, j} a_{j} \approx\left\{\begin{array}{cr}
\sum_{j=1}^{n_{\mathrm{n}}}\left(L_{i}\right)^{-1} \delta_{i, j} a_{j} & \left(\text { If } \mathrm{Fr}_{i}^{2}>1\right) \\
2\left(L_{i}\right)^{-1} & a_{i}-\sum_{j=1}^{n_{\mathrm{n}}} M_{i, j}\left(L_{i} L_{j}\right)^{-1} a_{j} \text { (Otherwise) }
\end{array}\right.
$$

with the fully-lumped mass matrix

$$
L_{i}=\sum_{j=1}^{n_{\mathrm{n}}} M_{i, j}
$$

where $\delta_{i, j}$ is the Kronecker's Delta and $\mathrm{Fr}_{i}^{2}$ is the maximum value of the local Froude number in the elements that share the $i$ th node. The second row of Eq.(7) serves as a perturbation of its left hand side, which converges to the non-lumped counterpart for a fine computational mesh (Guermond and Pasuquetti, 2013). According to Eq.(7), the mass matrix $\mathbf{M}$ is fully-lumped for supercritical flows while is not lumped for critical and subcritical flows as suggested in the first paragraph of this sub-section.

The mass matrix $\mathbf{M}$ is not lumped in the FEVM while is fully-lumped in the DFVM. The FEVM inverts $\mathbf{M}$ with the Gauss-Seidel method (Knabner and Angermann, 2003). The difference among the three numerical methods is only the treatment of $\mathbf{M}$, which critically affects behaviour of numerical solutions as shown in the next section. Under same boundary conditions and an initial guess, steady numerical solutions computed with the three numerical methods therefore coincide if they are obtained. Application of the mass matrix inversion method to $\mathbf{M}$ completely avoids the use of time consuming iterative methods at each time step, significantly improving computational efficiency over the FEVM. Preliminary numerical tests not presented here showed that a same level of computational efficiency is achieved in the DFVM and SELUM.

Temporal integration of each numerical method is carried out with the classical, fourth-order accurate Runge-Kutta method as in the previous researches (Unami and Alam, 2012; Yoshioka and Unami, 2012). The time increment $\Delta t$ is chosen sufficiently small so that the Courant-Friedrichs-Lewy condition (Toro, 2010) holds.

\subsection{Some remarks}

The three numerical methods, the SELUM, FEVM and DFVM, share a same discretization procedure except for the treatment of the mass matrix $\mathbf{M}$. As indicated in the literatures, treatment of a mass matrix controls errors in a temporal discretization (Christon et al., 2004; Guermond and Pasuquetti, 2013) and is one of the most influential factors in simulating time-dependent PDEs. A non-lumped mass matrix has higher phase and amplitude accuracy than the lumped counterpart, reducing numerical dissipation effectively; however, its solution may contain numerical oscillations for the problems with sudden transitions due to the use of the non-diagonal mass matrix whose inverse is not necessarily positive-definite (Harai, 2007). One consequence of this property is instability in simulating wet and dry interfaces for the flows with non-rectangular cross-sections in particular (Yoshioka et al., 2012). The use of a fully-lumped mass matrix does not encounter this kind of problems, but its solution is more dissipative than that of the non-diagonal counterpart. Comparisons of the lumped and non-lumped numerical methods for the 1-D and 2-D SWEs were carried out for some idealized cases (Szpilka and Kolar, 2003; Le Roux et al., 2011), but only a few have focused on real problems (Maßmann et al., 2010).

Several selective lumping algorithms for the 1-D and 2-D SWEs were proposed in the literatures (Kawahara et al., 1982; Yue, 1989) and have been applied to flood analyses (Yoshinaga, 2007; Kiri, 2012). However, these algorithms carry out the lumping procedure with a globally constant parameter whose optimal value needs to be determined a priori. Another drawback is the globality of the parameter that prevents to develop a flexible algorithm. The SELUM proposed in this paper is advantageous compared with the conventional ones because it has no parameter to be determined a priori and selectively lumps the mass matrix considering local flow conditions. Similar selective lumping algorithms have successfully been applied to linear and nonlinear parabolic PDEs (Yoshioka et al., 2013a; 2013b).

\section{Numerical analysis}

The SELUM is applied to three problems in order to see its performance for the flows with transitions or/and wet and dry interfaces that the FEVM or DFVM fails. The problems examined are an idealized dry dam break problem in a triangular channel (Sanders, 2001), experimental dry dam break problems with a triangular bump (Goutal and Maurel, 1997) and a hydraulic bore propagating upstream a rectangular flume (Unami and Alam, 2012). Analytical or experimental results are available for these problems.

\subsection{Dry dam break problem in a triangular channel}

An idealized dam break problem in a flat, frictionless triangular channel is firstly considered. Sanders (2001) investigated dam break problems in non-rectangular channels followed up on by several researchers (Ying et al., 2004; 
Rosatti, 2011). Shallow water flows in triangular channels are generally difficult to accurately compute compared with those in rectangular ones because the top width of the cross-section varies in direct proportion to the water depth, significantly reducing accuracy and stability of a numerical method for flows on a dry bed in particular.

Here a 2,000 (m) length flat straight channel is considered as the computational domain $(0,2000)$ (m). The initial water elevation $\eta(\mathrm{m})$ is set as

$$
\eta= \begin{cases}h_{\mathrm{U}} & (x \leq 1000) \\ h_{\mathrm{D}} & (x>1000)\end{cases}
$$

where $h_{\mathrm{U}}$ and $h_{\mathrm{D}}$ denote the initial upstream and downstream water depths in the channel, respectively. The initial discharge is set as $Q=0 \quad\left(\mathrm{~m}^{3} / \mathrm{s}\right)$ in the entire channel. The initial water depths are set as $h_{\mathrm{U}}=10(\mathrm{~m})$ and $h_{\mathrm{D}}=0$ (m). Both the upstream and downstream boundaries of the channel are solid walls. Analytical solution to this problem is presented in Chen et al. (2011). The solution involves a wet and dry interface, which is difficult to accurately capture even for recently developed high-resolutions methods (Venutelli, 2006; Ouyang et al., 2013).

The channel is uniformly discretized into a computational mesh with the elemental length of $\Delta x$. The following two cases of $\Delta x=10 \quad(\mathrm{~m})\left(n_{\mathrm{e}}=200\right)$ and $\Delta x=1 \quad(\mathrm{~m})$ $\left(n_{\mathrm{e}}=2,000\right)$ are investigated. The time increment $\Delta t$ is fixed to 0.001 (s). In this problem, an improved upwinding method for evaluation of the elemental momentum flux is applied to the numerical methods in order to sharply capture the wet and dry interface (Yoshioka et al., 2013e). The FEVM fails to give numerical solutions to this problem under the stated conditions because of the instability encountered at the wet and dry interface.

Computed water surface profiles at $t=30$ (s) for the DFVM and SELUM are illustrated in Figures. 1 and 2. The obtained results are sufficiently accurate reproducing well the rarefaction wave for both of $\Delta x$. The water surface profiles of the DFVM monotonically decrease toward the downstream being consistent with the analytical solution, while those of the SELUM involve small oscillations around the upstream-side of the rarefaction. These oscillations are due to the non-diagonal nature of the mass matrix $\mathbf{M}$ in the SELUM, which disappear as the mesh is refined.

\subsection{Dry dam break problems with a triangular bump}

Experimental dam break problems with bed topography are next considered. The experimental setting is shown in Figure 3. The channel has a uniformly rectangular cross-section with the width of $1.75(\mathrm{~m})$, and consists of an upstream reservoir filled up by water with the depth of 0.75 (m) and a dry downstream channel having a triangular bump with the height of $0.40(\mathrm{~m})$. A vertical wall serving as a dam is installed at $15.5(\mathrm{~m})$, which is assumed to be instantaneously removed at the initial time $t=0$ (s). The Manning's coefficient $n_{\mathrm{M}}$ of the channel has been estimated as $0.0125\left(\mathrm{~s} / \mathrm{m}^{1 / 3}\right)$ (Liang and Marche, 2009). The

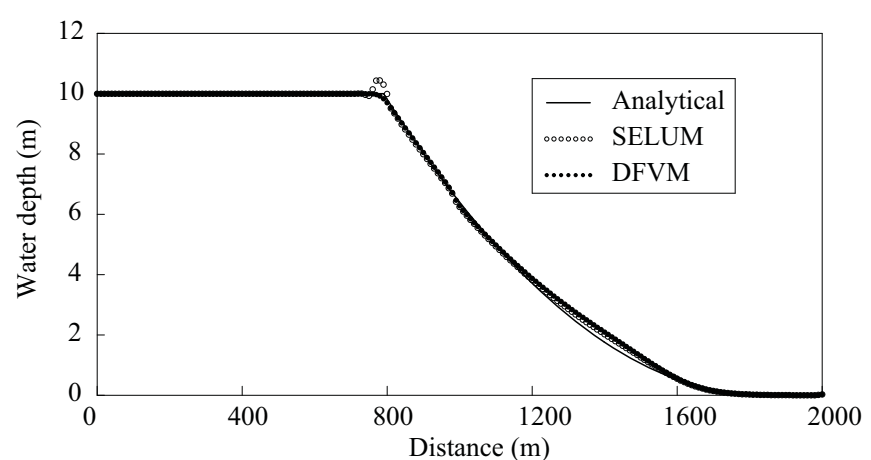

Figure 1: Comparisons of the computed water surface profiles with the elemental length of $\Delta x=10 \quad(\mathrm{~m})$

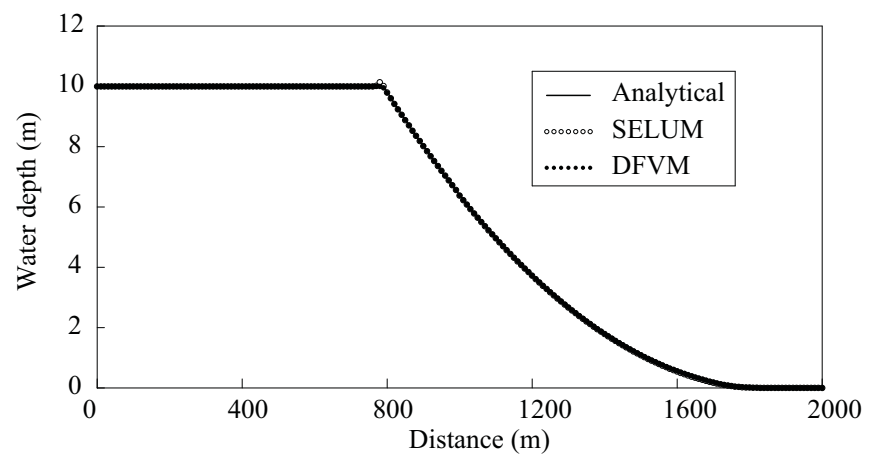

Figure 2: Comparisons of the computed water surface profiles with the elemental length of $\Delta x=1 \quad(\mathrm{~m})$

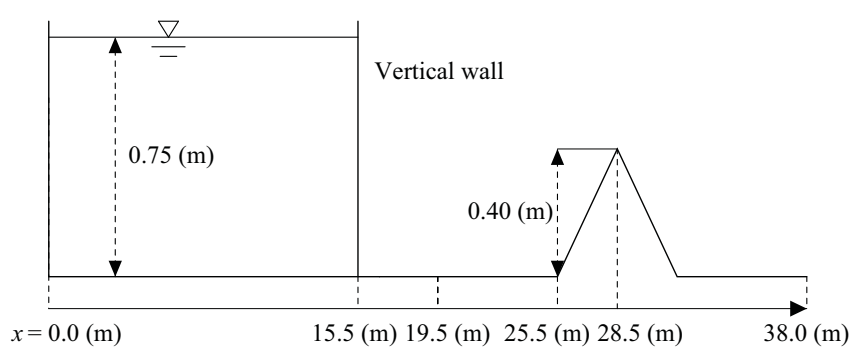

Figure 3: Sketch of the experimental channel for dry dam break problems with a triangular bump

upstream-end of the channel is a solid wall. Here two cases of the problems, which are Cases A and B, are considered. In Case $A$, free outflow of water occurs at the downstream-end of the channel. In Case B, the downstream-end is a solid wall and the area downstream of the bump is filled with a still water whose water depth equals to 0.15 (m). Water depths were measured at several points in the channel for both of the cases (Goutal and Maurel, 1997). Water flows of the presented cases involve a wet and dry interface moving over the bump and receding bores created when the discharged waters from the reservoir hit the triangular bump and the solid wall (Case B). These cases have served as severe benchmarks for numerical methods to examine the ability to handle transcritical flows with wet and dry interfaces on irregular topography (Aricò and Tucciarelli, 2007; Singh et al., 2011; Aricò et al., 2013; Liu et 
(a) DFVM

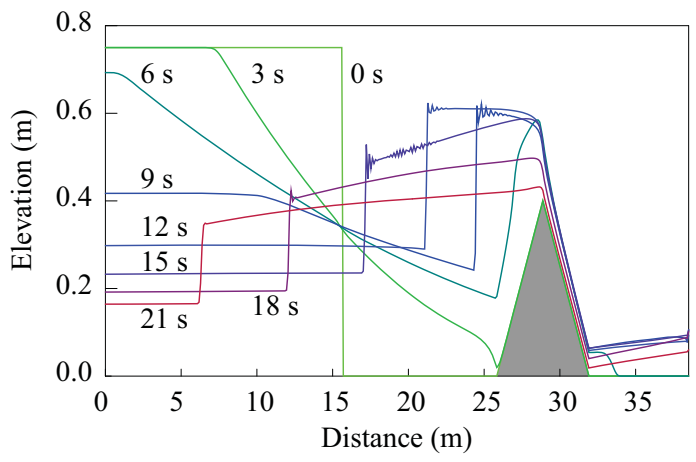

(b) SELUM

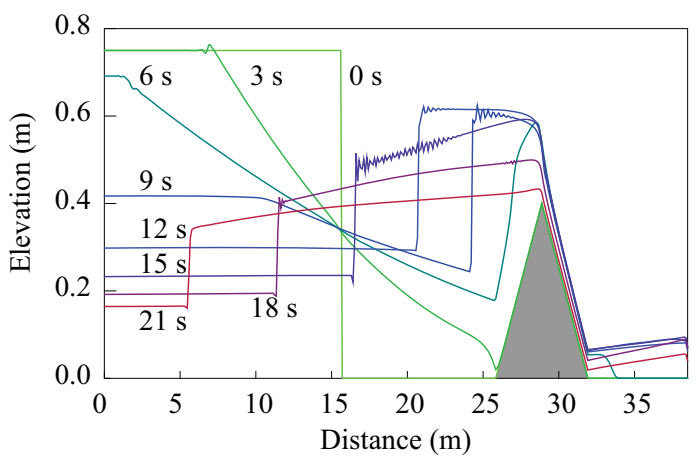

Figure 4: Computed water surface profiles in the experimental channel for Case A

(a) DFVM

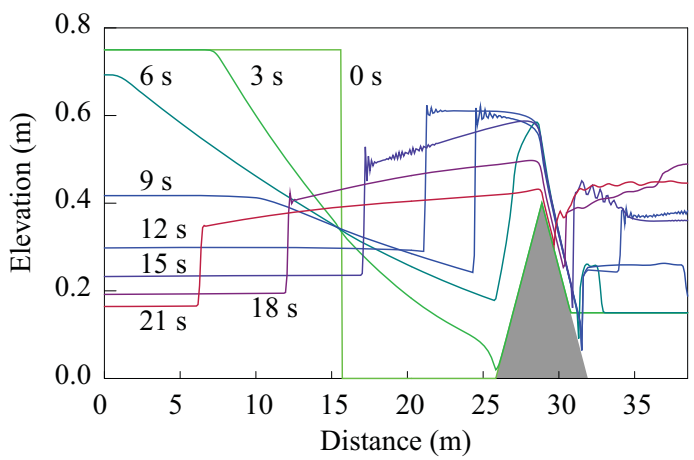

(b) SELUM

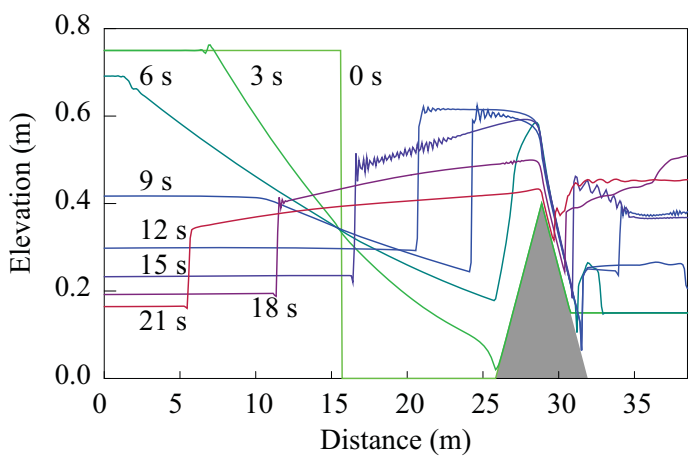

Figure 5: Computed water surface profiles in the experimental channel for Case B

al., 2013).

The channel is divided into $n_{\mathrm{e}}=380$ uniform elements. The time increment is $\Delta t=0.004$ (s). The FEVM fails under the stated conditions due to an instability encountered when a wet and dry interface approaches the bump. The DFVM and SELUM are therefore applied to these problems.

Figures 4 and 5 show computed water surface profiles for the DFVM and SELUM in Cases A and B, respectively. The figures demonstrate that both the DFVM and SELUM sharply capture the receding bores. The results of the SELUM involve small oscillations around the upstream-side of the rarefaction as in the previous problem. Other oscillations in the water surface profiles are due to the absence of slope limiting procedures in the numerical methods.

Figures 6 and 7 show comparisons of the computed and measured water depths in Cases A and B at $x=19.5$ (m) (between the dam and the bump), $x=25.5$ (m) (upstream-end of the bump), $x=28.5$ (m) (top of the bump) and $x=35.5(\mathrm{~m})$ (downstream of the bump). Computational results for the DFVM and SELUM in Figures 6 and 7 are comparable to those of the high-resolution methods (Bollermann, 2013; Guan et al., 2013) despite that they utilize a simpler spatial discretization. The DFVM and SELUM are almost equally accurate for both of the Cases. Errors between the computed and measured results are mainly due to the hydrostatic pressure assumption of the 1-D SWEs and turbulent nature of the flows (Rebollo et al., 2003). According to Figures 6 and 7, computed wave prop- agation speed of the SELUM is slightly faster than that of DFVM, resulting in the earlier risings of the water depths. Although these differences become small for finer computational meshes, the results indicate significance of the treatment of the temporal term for some practical problems, such as risk analysis of a flash flooding in which estimation of flood peaks is of importance (Yoshioka et al., 2013d).

\section{3 hydraulic bore propagating upstream}

The third problem considered is a hydraulic bore propagating upstream, a moving shock on a uniform supercritical flow. A laboratory experiment in a 20 (m) long flume having a rectangular cross-section with the width of 0.60 (m) and the bed slope of 0.01 was carried out. A uniform supercritical flow with the water depth of $0.026(\mathrm{~m})$ and the discharge of $0.01295\left(\mathrm{~m}^{3} / \mathrm{s}\right)$ was set up in the flume. Froude number of the flow was 1.64. Downstream-end of the flume was suddenly closed at the time $t=0$ (s) to create a bore propagating upstream. Front position of the bore was estimated from a video image as

$$
t=\left(0.2137 x^{*}+1.9344\right) x^{*}
$$

where $x^{*}$ is the front position of the bore measured from the downstream-end of the flume. The Manning's coefficient $n_{\mathrm{M}}$ of the flume is estimated as $0.01\left(\mathrm{~s} / \mathrm{m}^{1 / 3}\right)$. The entire flume is discretized into a computational mesh with $n_{\mathrm{e}}=2,000$ elements. The time increment is $\Delta t=0.01$ (s). Unami and Alam (2012) demonstrated that the FEVM 


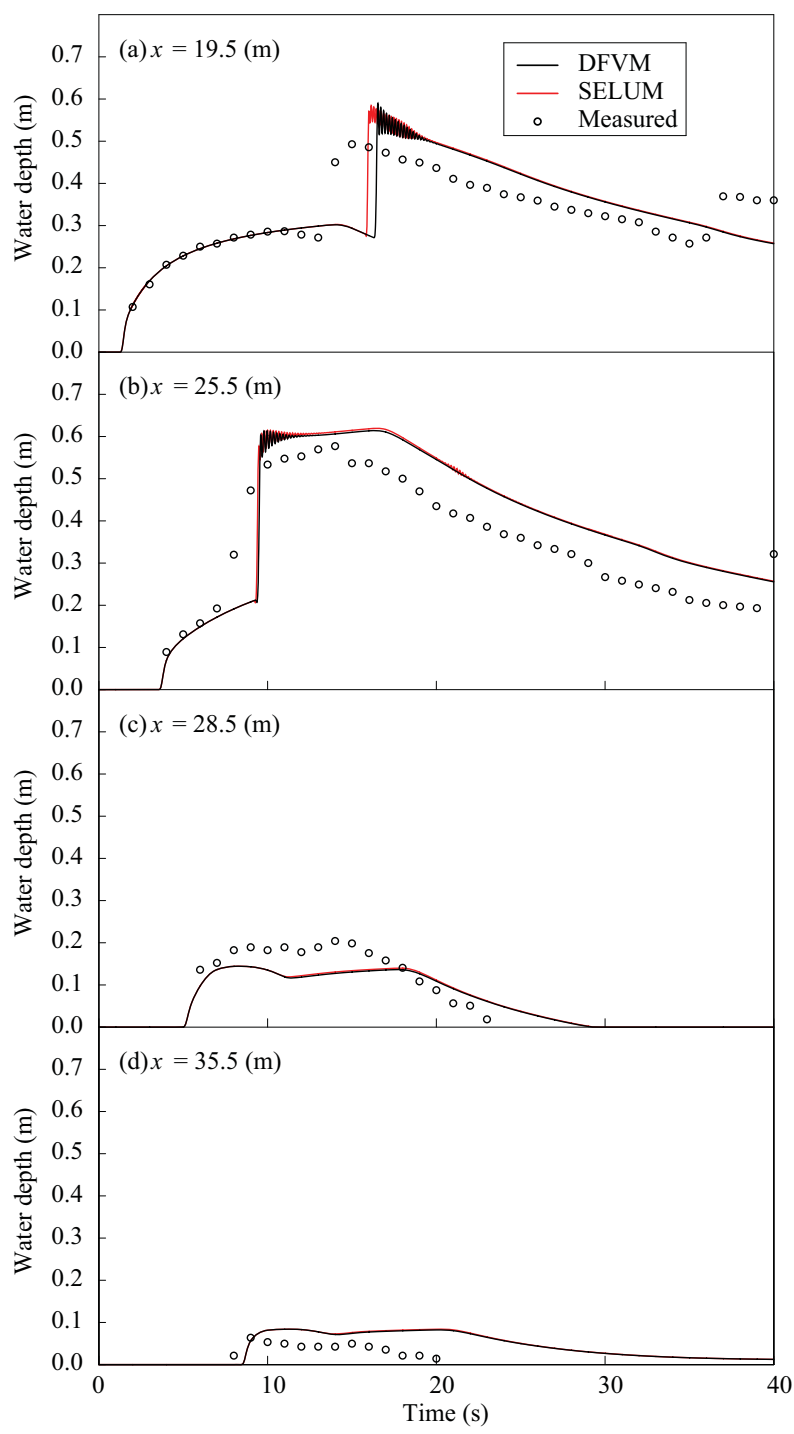

Figure 6: Comparisons of the water depth time series for Case A accurately simulates the hydraulic bore in the flume, capturing the celerity of the shock front, while Yoshioka et al. (2012) showed that the DFVM fails causing large oscillations at the downstream-end of the flume, which lead to negative water depths. The FEVM and SELUM are therefore applied to this problem.

Figure 8 shows computed water surface profiles for the FEVM and SELUM with the time interval of 10 (s). Also plotted in the figure are observed front positions of the bore based on Eq.(10). Both of the numerical methods accurately reproduce the hydraulic bore capturing its sharp front. Propagation speed of the bore is faster in the SELUM, resulting in relatively higher accuracy in predicting the front position of the bore. Differences between the water surface profiles of the two numerical methods become smaller as the time elapses because of the frictional effect. Computational time of the SELUM for this problem is less than half of that of the FEVM, demonstrating its higher efficiency.

\section{Conclusions}

Temporal discretization procedure is a crucial part in simulating transient flows with the 1-D SWEs. This paper de-

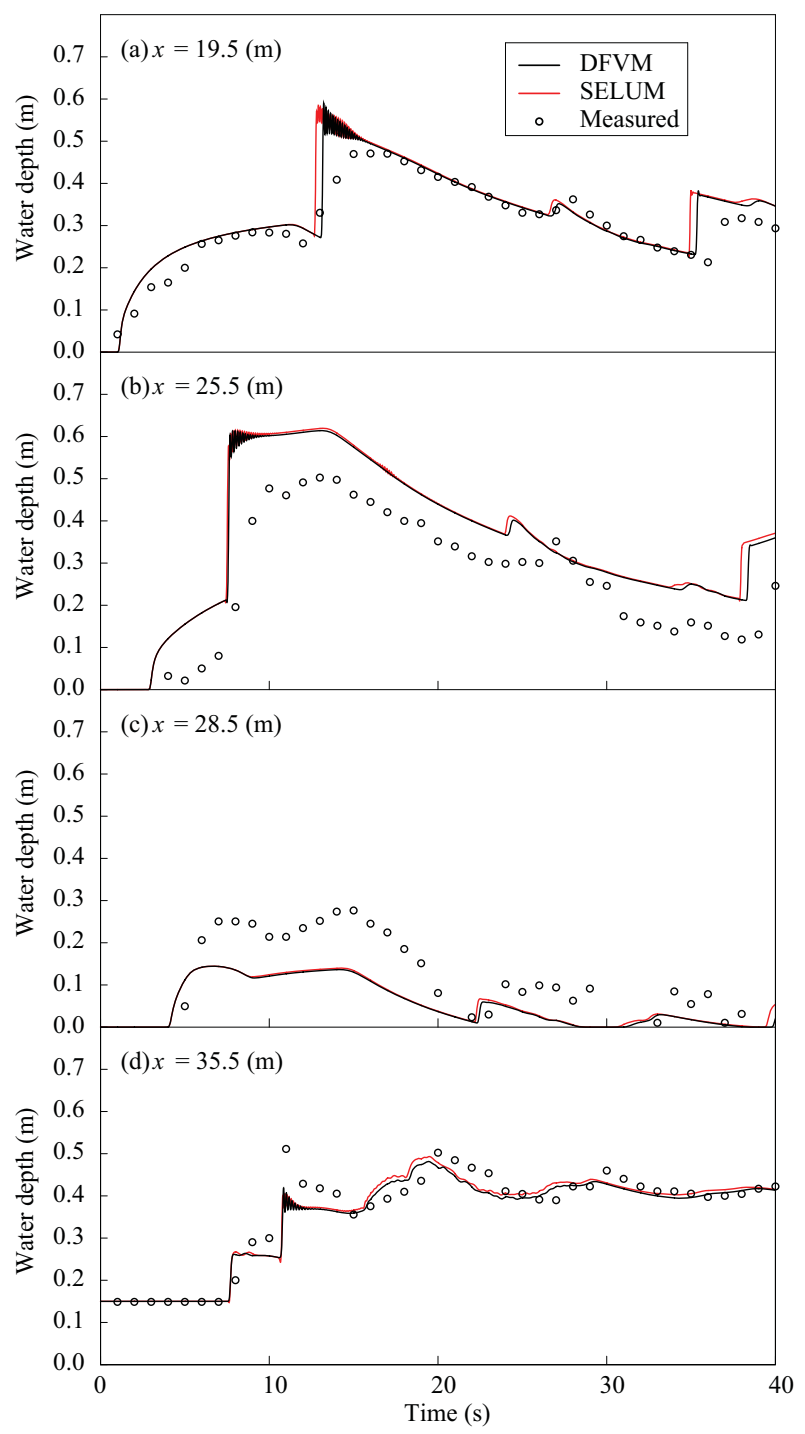

Figure 7: Comparisons of the water depth time series for Case B

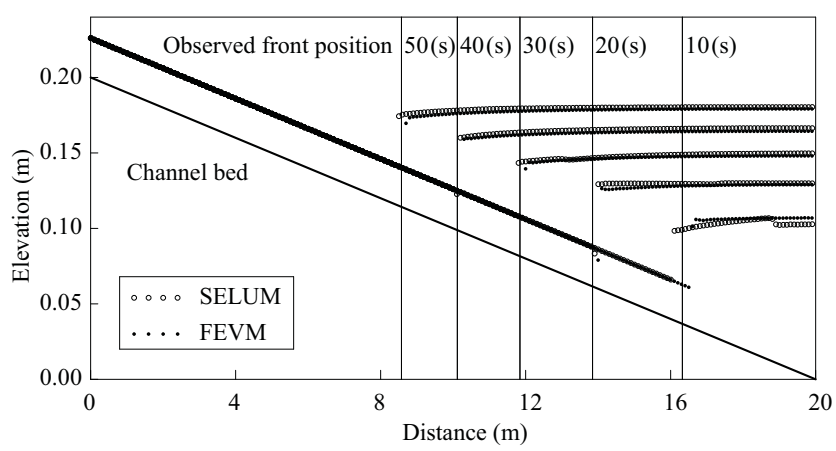

Figure 8: Computed water surface profiles for the hydraulic bore

veloped the SELUM, a new numerical method for the 1-D SWEs that selectively lumps the temporal term of the continuity equation in order to efficiently compute accurate and stable solutions. Performance of the SELUM was compared with those of the FEVM and DFVM, which are the non-lumped and fully-lumped counterparts, respectively. Computational results of the idealized and experimental problems demonstrated that the SELUM accurately handles the flows that the FEVM or DFVM fails. The approximate 
mass matrix inversion method used in the SELUM contributed to its higher computational efficiency than the FEVM. A drawback of the SELUM is that it does not preserve monotonic water surface profiles of the rarefaction waves. However, it was also shown that this does not significantly affect overall accuracy of the numerical solutions. Because of the spatial discretization procedure suited to solve flows in open channel networks, application of the SELUM to such flows is considered not to encounter technical difficulties. Detailed investigation of the SELUM for transient flows in open channel networks will be carried out elsewhere.

This paper focused on implementation of the selective lumping algorithm to the 1-D SWEs in single channels. In practical analysis, the 2-D SWEs are also in wide use. Since numerical simulation of the 2-D SWEs is computationally more demanding than the 1-D counterpart in general, the lumping algorithm analogous to the SELUM is considered to serve as an effective way to obtain accurate numerical solutions without significantly increasing computational costs. Its application to other PDEs in hydraulics and related research fields is also an important research topic.

\section{Acknowledgement}

This study is financially supported by the JSPS under grant $25 \cdot 2731$.

\section{References}

[1] Aricò, C., and Tucciarelli, T. (2007): A marching in space and time (MAST) solver of the shallow water equations. Part I: The 1D model, Adv. Water Resour., 30(5), pp.1236-1252.

[2] Aricò, C., Sinagra, M., and Tucciarelli, T. (2013): Anisotropic potential of velocity fields in real fluids: Application to the MAST solution of shallow water equations, $A d v$. Water Resour., 62(Part A), pp.13-26.

[3] Begnudelli, L., and Sanders, B.F. (2007): Simulation of the St. Francis dam-break flood. J. Engrg. Mech. 133(11), pp.1200-1212.

[4] Bollermann, A., Chen, G., Kurganov, A., and Noelle, S., (2013): A well-balanced reconstruction of wet/dry fronts for the shallow water equations, J. Sci. Comput., 56(2), pp.267-290.

[5] Boscheri, W., Dumbser, M., and Righetti, M. (2012) A semi-implicit scheme for $3 \mathrm{D}$ free surface flows with high-order velocity reconstruction on unstructured Voronoi meshes, Int. J. Numer. Methods Fluids, 72(6), pp.607-631.

[6] Boudarias, C., and Gerbi, S. (2007): A finite volume scheme for a model coupling free surface and pressurised flows in pipes, J. Comput. Appl. Math., 207(1), pp.109-131.

[7] Chen, Y.C., Wu, B., and Wang, B. (2011): Similarity solution of dam-break flow on horizontal frictionless channel, $J$. Hydraul. Res., 49(3), pp.384-387.

[8] Christon, M.A., Martinez M.J., and Voth, T.E. (2004): Generalized Fourier analyses of the advection-diffusion equation-Part I: One-dimensional domains, Int. J. Numer. Methods Fluids, 45(8), pp.839-887.

[9] Cunge, J.A., Holly Jr., F.M., and Verwey, A. (1980): Practical Aspects of Computational River Hydraulics, Pitman, pp.7-52.

[10] Delestre, O., Lucas, C., Ksinant, P.A., Darboux, F., La- guerre, C., Vo, T.N.T., James, F., and Cordier, S. (2013): SWASHES: a compilation of shallow water analytic solutions for hydraulic and environmental studies, Int. J. Numer. Methods Fluids, 72(3), pp.269-320.

[11] Dumbser, M. (2013): A diffuse interface method for complex three-dimensional free surface flows, Comput. Methods Appl. Mech. Engrg., 253(1), pp.186-198.

[12] Dumbser, M., and Casulli, V. (2013): A staggered semi-implicit spectral discontinuous Galerkin scheme for the shallow water equations, Appl. Math. Comput., 219(15), pp.8057-8077.

[13] Goutal, N., and Maurel, F. (1997): Proc. of the 2nd Workshop on Dam-break Wave Simulation, EDF-DER Report, HE-43/97/016/B.

[14] Goutal, N., and Santie-Marie, J. (2011): A kinetic interpretation of the section-averaged Saint-Venant system for natural river hydraulics, Int. J. Numer. Methods Fluids, 67(7), pp.914-938.

[15] Guan, M., Wright, N.G., and Sleigh, P.A. (2013): A robust 2D shallow water model for solving flow over complex topography using homogenous flux method, Int. J. Numer. Methods Fluids, 73(3), pp.225-249.

[16] Guermond, J.L., and Pasuquetti, R. (2013): A correction technique for the dispersive effects of mass lumping for transport problems, Comput. Methods Appl. Mech. Engrg., 253(1), pp.186-198.

[17] Harai, I., and Hauke, G. (2007): Semidiscrete formulation for transient transport at small time steps, Int. J. Numer. Methods Fluids, 54(6-8), pp.731-743.

[18] Hodges, B.R. (2013): Challenges in continental river dynamics, Environ. Model. Software, 50, pp.16-20.

[19] Kawahara M., Hirano H., and Tsubota K. (1982): Selective lumping finite element method for shallow water flow, Int. J. Numer. Methods Fluids, 2(1), pp. 89-112.

[20] Knabner, P., and Angermann, L. (2003): Numerical Methods for Elliptic and Parabolic Partial Differential Equations, Springer-Verlag, pp.283-315.

[21] Kiri, H. (2012): Study for developing a storm surge flood model in coastal farmland, Technical Report of NARO, 51, pp.109-164.

[22] Le Roux, D.Y., Dieme, M., and Sene, A. (2011): Time discretization schemes for Poincaré waves in finite-element shallow-water model, J. Sci. Comput., 33(5), pp.2217-2246.

[23] Liang, Q., and Marche, F. (2009): Numerical resolution of well-balanced shallow water equations with complex source terms, Adv. Water. Resour., 32(6), pp.873 -884.

[24] Liu, Y., Zhou, J., Song, L., Zou, O., Liao, L., and Wang, Y. (2013): Numerical modelling of free-surface shallow flows over irregular topography with complex geometry, Appl. Math. Model., 37(23), pp.9482-9498.

[25] Malleron, N., Zaoui, F., Goutal, N., and Morel, T. (2011): On the use of a high-performance framework for efficient model coupling in hydrodynamics, Environ. Model. Software, 26(12), pp.1747-1758.

[26] Maßmann, S., Androsov, A., and Danilov, V. (2010): Intercomparison between finite element and finite volume approaches to model North Sea tides, Cont. Shelf. Res., 30(6), pp.680-691.

[27] Marras, S., Moragues, M., Vázquez, M., Jorba, O., and Houzeaux, G. (2013): Simulations of moist convection by a variational multiscale stabilized finite element method, $J$. Comput. Phys., 252, pp.195-218. 
[28] Mishev, I.D. (1998): Finite volume methods on Voronoi meshes, Numer. Methods Partial Differ. Equat., 14(2), pp.193-212.

[29] Ono, K., Kazama, S., Kawagoe, S., and Yokoo, Y. (2011): Dam break caused by the 2011 off the Pacific Coast of Tohoku Earthquake, Fukushima Prefecture, Japan. J. JSNDS., 30(3), pp.359-367, (in Japanese).

[30] Osher, S., and Solomon, F. (1982): Upwind difference schemes for hyperbolic systems of conservation laws, Math. Comput., 38(158), pp.339-374.

[31] Ouyang, C., He, S., Xu, Q., Luo, Y., and Zhang, M. (2013) A MacCormack-TVD finite difference method to simulate the mass flow in mountainous terrain with variable computational domain, Comput. Geosci., 52, pp.1-10.

[32] Rebollo, T.C., Nieto, F.D.F., and Marmol, M.G. (2003): A flux-splitting solver for shallow water equations with source terms, Int. J. Numer. Methods Fluids, 42(2), pp.23-55.

[33] Ricchiuto, M., and Bollermann, A. (2009): Stabilized residual distribution for shallow water simulations, J. Comput. Phys., 228(4), pp.1071-1115.

[34] Roe, P. (1981): Approximate Riemann solvers, parameter vectors, and difference schemes, J. Comput. Phys., 43(2), pp.357-372.

[35] Roggensack, A. (2012): A kinetic scheme for the one-dimensional open channel flow equations with applications on networks, Calcolo, 50(4), pp.255-282.

[36] Rosatti, G., Bonaventura, L., Deponti, A., and Garegnani, G. (2011): An accurate and efficient semi-implicit method for section-averaged free-surface flow modeling. Int. J. Numer. Methods Fluids, 65(4), pp.448-473.

[37] Sármány, D., Hubbard, M.E., and Ricchiuto, M. (2013): Unconditionally stable space-time discontinuous residual distribution for shallow-water flows, J. Comput. Phys., 253, pp.86-113.

[38] Sanders, B.F. (2001): High-resolution and non-oscillatory solution for the St Venant equations in non-rectangular and non-prismatic channels. J. Hydraulic Res. 39(3), pp.321-330.

[39] Singh, J., Altinakar, M.S., and Ding, Y. (2011): Two-dimensional numerical modeling of dam-break flows over natural terrain using a central explicit scheme, $A d v$. Water. Resour., 34(10), pp.1366-1375.

[40] Spizlka, C.M., and Kolar, R.L. (2003): Numerical analogous to Fourier and dispersion analysis: development, verification, and application to the shallow water equations, Adv. Water Resour., 26(6), pp.649-662.

[41] Stoker, J.J. (1957): Water waves, Interscience publishing, New York, pp.291-341.

[42] Szymkiewicz, R. (2010): Numerical Modeling in Open Channel Hydraulics, Springer, pp.263-300.

[43] Toro, E.F. (2010): Riemann Solvers and Numerical Methods for Fluid Dynamics, Springer, pp.163-212.

[44] Tumoro, G., Bonaventura, L., and Restelli, M. (2013): A semi-implicit, semi-Lagrangian, $p$-adaptive discontinuous Galerkin method for the shallow water equations, J. Comput. Phys., 232(1), pp.46-67.

[45] Unami, K., and Alam, A.H.M.B. (2012): Concurrent use of finite element and finite volume methods for shallow water equations in locally 1-D channel networks, Int. J. Numer.
Methods Fluids, 69(2), pp.255-272.

[46] Voth, T.E., Christon, M.A., and Martinez M.J. (2004): Generalized Fourier analyses of the advection-diffusion equation-Part II: Two-dimensional domains, Int. J. Numer. Methods Fluids, 45(8), pp.889-920.

[47] Vacondio, R., Rogers, B.D., Stanby, P.K., and Mignosa, P. (2013): Shallow water SPH for flooding with dynamic particle coalescing and splitting, Adv. Water Resour., 59, pp.10-23.

[48] Venutelli, M. (2006): A third-order explicit central scheme for open channel flow simulations, J. Hydraul. Res., 44(3), pp.402-411.

[49] Xia, X., Liang, Q., Pastor, M., Zou, W., and Zhuang, Y.F. (2013): Balancing the source terms in a SPH model for solving the shallow water equations, Adv. Water Resour., 59, pp.25-38.

[50] Ying, X., Khan, A.A., and Wang, S.S.Y. (2004): Upwind conservative schemes for the Saint Venant equations. $J$. Hydraul. Eng., 130(10), pp.977-987.

[51] Yen, B.C. (2002): Open channel flow resistance, J. Hydraul. Eng, 128(1), pp.20-39.

[52] Yoshinaga, I. (2007): Studies on hydraulic and biochemical phenomena of an irrigation reservoir and ponded water in a paddy field, Technical Report of NARO, 47, pp.1-48.

[53] Yoshioka, H., and Unami, K. (2012): A dual grid based finite volume scheme for analysis of shallow water flows in locally one-dimensional open channel network flows, Proceedings of the $69^{\text {th }}$ Japanese Society of Irrigation, Drainage and Rural Engineering, Kyoto Division, pp.114-115, (in Japanese).

[54] Yoshioka, H., Unami, K., and Fujihara M. (2012): On temporal discretization of the one-dimensional shallow water numerical model, Proc. the Annual Meeting of Applied Hydraulics in 2012, pp. 96-100, (in Japanese).

[55] Yoshioka, H., Kinjo, N., Unami, K., and Fujihara, M. (2013a): Application of a conforming Petrov-Galerkin finite element scheme to analysis of longitudinal dispersion phenomena in open channel networks, Proc. $35^{\text {th }}$ IAHR WORLD CONGRESS, Paper No. 10491, 12p.

[56] Yoshioka, H., Kinjo, N., Unami, K., and Fujihara, M. (2013b): A conforming finite element method for non-conservative advection-diffusion equations on connected graphs, J. JSCE., Ser. A2 (Applied Mechanics), 69(2), pp. I_59-I_70, (in Japanese).

[57] Yoshioka, H., Takagi, K., Unami, K., and Fujihara, M., (2013c): A horizontally 2-D shallow water model utilizing both the finite element and the finite volume techniques, Proc. the $70^{\text {th }}$ JSIDRE, Kyoto Div., 2p, (in Japanese).

[58] Yoshioka, H., Unami, K., and Fujihara, M. (2013d): A dual grid based finite volume model for dam break flows in multiply connected open channel networks with general cross-sections, Proc. $62^{\text {nd }}$ National Congress Theor. Appl. Mech., OS19-09, 2pp, (in Japanese).

[59] Yoshioka, H., Unami, K., and Fujihara, M. (2013e): A simple finite volume model for dam break problems in multiply connected open channel networks with general cross-sections, Theor. Appl. Mech. Japan. (in press)

[60] Yue, J. (1989): Selective lumping effects on depth-integrated finite element model of channel flow, $A d v$. Water Resour., 12(2), pp.74-78. 